\title{
2D shear wave liver elastography by Aixplorer to detect portal hypertension in cirrhosis: An individual patient data meta- analysis
}

\author{
Maja Thiele 1,2 (D) | Mie B. Hugger ${ }^{1,2}$ (D) | Yongsoo Kim ${ }^{3}$ | Pierre-Emmanuel Rautou ${ }^{4,5,6}$ | \\ Laure Elkrief ${ }^{4,5,6}$ | Christian Jansen ${ }^{7}$ | Wim Verlinden ${ }^{8}$ | Giulia Allegretti ${ }^{9}$ | \\ Mads Israelsen $^{1,2}$ | Horia Stefanescu ${ }^{10}$ | Fabio Piscaglia9 (i) | Juan C. García-Pagán ${ }^{11}$ \\ Sven Franque $^{8}$ | Annalisa Berzigotti ${ }^{12}$ (i) | Laurent Castera ${ }^{4,13}$ | Woo K. Jeong ${ }^{14}$ | \\ Jonel Trebicka ${ }^{1,7,12,15}$ (i) | Aleksander Krag ${ }^{1,2}$ \\ ${ }^{1}$ Department of Gastroenterology and Hepatology and OPEN Patient Data Explorative Network, Odense University Hospital, Odense, Denmark \\ ${ }^{2}$ Department of Clinical Research, University of Southern Denmark, Odense, Denmark \\ ${ }^{3}$ Department of Radiology, College of Medicine, Hanyang University Guri Hospital, Gyeonggi-do, Korea \\ ${ }^{4}$ Centre de recherche sur l'inflammation, Inserm, Université de Paris, Paris, France \\ ${ }^{5}$ Service d'Hépatologie, DMU Digest, Hôpital Beaujon, AP-HP, Clichy, France \\ ${ }^{6}$ Centre de Référence des Maladies Vasculaires du Foie, French Network for Rare Liver Diseases (FILFOIE), European Reference Network (ERN) 'Rare-Liver', \\ Paris, France \\ ${ }^{7}$ Department of Internal Medicine I, University of Bonn, Bonn, Germany \\ ${ }^{8}$ Department of Gastroenterology Hepatology, University Hospital Antwerp \& Translational Sciences in Inflammation and Immunology TWI2N, Faculty of \\ Medicine and Health Sciences, University of Antwerp, Antwerp, Belgium \\ ${ }^{9}$ Unit of Internal Medicine, Hospital S.Orsola-Malpighi, University of Bologna, Bologna, Italy \\ ${ }^{10}$ Liver Unit, Regional Institute of Gastroenterology and Hepatology, Cluj-Napoca, Romania \\ ${ }^{11}$ Barcelona Hepatic Hemodynamic Laboratory, Liver Unit, Hospital Clínic, Institut de Investigacions Biomèdiques August Pi i Sunyer (IDIBAPS) and CIBEREHD, \\ University of Barcelona, Barcelona, Spain \\ ${ }^{12}$ Hepatology, UVCM, Inselspital, University of Bern, Bern, Switzerland \\ ${ }^{13}$ Hepatogastroenterology Unit, CHU de Tours, Tours, France \\ ${ }^{14}$ Department of Radiology, Center for Imaging Science, Samsung Medical Center, Sungkyunkwan University School of Medicine, Seoul, Korea \\ ${ }^{15}$ Translational Hepatology, Medical Department I, Goethe University Clinic, Frankfurt, Germany \\ the
}

\section{Correspondence}

Maja Thiele, Department of

Gastroenterology and Hepatology, Odense University Hospital, Kloevervaenget 10,

entrance 112, 11th floor, DK-5000 Odense

C, Denmark.

Email: maja.thiele@rsyd.dk

Funding information

Maja Thiele received funding from the

Region of Southern Denmark's postdoc

\begin{abstract}
Background \& Aims: Liver stiffness measured with 2-dimensional shear wave elastography by Supersonic Imagine (2DSWE-SSI) is well-established for fibrosis diagnostics, but non-conclusive for portal hypertension.
\end{abstract}


funds. Mie Balle Hugger received funding from the Odense University Hospital Fund for Pre-graduate Stipends. Data collected in Bologna were partially supported by the 2013 People Progamme, a Marie Curie Action under the European Commission's Seventh Framework Programme (FP7PEOPLE-2013-IAPP). Jonel Trebicka is supported by grants from Deutsche Forschungsgemeinschaft (grants SFB TRR57 and CRC 1382), the European Union's Horizon 2020 Research and Innovation Programme (Grant 668031 GALAXY), (Grant 825694 MICROB-PREDICT) and (Grant 731875 LIVERHOPE) as well as from the Cellex Foundation (grant PREDICT).

Handling Editor: Jian Sun and upper endoscopy as reference. We used meta-analytical integration of diagnostic accuracies with optimized rule-out (sensitivity-90\%) and rule-in (specificity-90\%) cut-offs.

Results: Five studies from seven centres shared data on 519 patients. After exclusion, we included 328 patients. Eighty-nine (27\%) were compensated and 286 (87\%) had CSPH. 2DSWE-SSI < $14 \mathrm{kPa}$ ruled out CSPH with a summary AUROC (sROC), sensitivity and specificity of $0.88,91 \%$ and $37 \%$, and correctly classified $85 \%$ of patients, with minimal between-study heterogeneity. The false negative rate was $60 \%$, of which decompensated patients accounted for 78\%. 2DSWE-SSI $\geq 32 \mathrm{kPa}$ ruled in CSPH with sROC, sensitivity, specificity and correct classifications of $0.83,47 \%, 89 \%$ and 55\%. In a subgroup analysis, the $14 \mathrm{kPa}$ cut-off showed consistent sensitivity and higher specificity for patients with compensated cirrhosis, without ascites, viral aetiology or BMI $<25 \mathrm{~kg} / \mathrm{m}^{2}$. 2DSWE-SSI ruled out severe portal hypertension and large varices with fewer correctly classified and lower sROC, and with minimal benefit for ruling in.

Conclusion: Liver stiffness using 2-dimensional shear wave elastography below $14 \mathrm{kPa}$ may be used to rule out clinically significant portal hypertension in cirrhosis patients, but this would need validation in populations of compensated liver disease. 2DSWE-SSI cannot predict varices needing treatment.

\section{KEYWORDS}

Aixplorer, compensated advanced chronic liver disease, Decompensated liver disease, liver vein catheterization, supersonic shear imagine

\section{1 | INTRODUCTION}

Chronic liver disease costs 39 million disability-adjusted life years annually, with decompensated cirrhosis driving excess morbidity and mortality. ${ }^{1}$ The consequence of a change from compensated to decompensated cirrhosis is most obvious in 5-year mortality rates, which increase from $1.5 \%$ in compensated cirrhosis to $88 \%$ in cirrhosis with multiple decompensating events. ${ }^{2}$ In compensated cirrhosis, the risk of developing varices, and the risk of decompensation, increases dramatically when the hepatic venous pressure gradient (HVPG) is $\geq 10$ or $\geq 12 \mathrm{mmHg}$, marking the thresholds for clinically significant portal hypertension (CSPH) and severe portal hypertension (SPH). ${ }^{3,4}$ Liver vein catheterization with measurement of HVPG is the diagnostic gold standard for the indirect portal pressure assessment in patients with cirrhosis, but the procedure is restricted to highly specialized centres. ${ }^{5}$ Therefore, non-invasive tools with high accuracy to rule in or rule out $\mathrm{CSPH}, \mathrm{SPH}$ and varices needing treatment in patients with cirrhosis are needed.

Transient elastography (TE) with FibroScan ${ }^{\circledR}$ (Echosens, France) alone or in combination with platelet count and/or spleen diameter is widely used for portal hypertension. ${ }^{6,7}$ TE below $20 \mathrm{kPa}$ is a key feature of the Baveno VI criteria to rule out varices needing treatment in patients with compensated advanced chronic liver disease. ${ }^{4}$ The applicability of TE is, however, limited in patients with ascites,

\section{Key points}

- This individual patient data meta-analysis combines data from 328 patients from five different studies, which have examined the role of 2-dimensional shear wave elastography by Supersonic Imagine (2DSWE-SSI) to detect clinically significant portal hypertension (CSPH) in patients with cirrhosis.

- We found that 2DSWE-SSI may be used to rule out CSPH due to a high summary AUROC (sROC) of 0.88 and sensitivity of $91 \%$, with minimal between-study heterogeneity.

- The existing body of evidence is limited by a substantial selection bias, as a high proportion of patients had decompensated cirrhosis at study inclusion. This impairs the generalizability of results from the meta-analysis.

narrow intercostal space, obesity or displaced liver, and has an upper measurement limit of $75 \mathrm{kPa} .^{8}$

A 2-dimensional real-time shear wave elastography (2DSWESSI) combines an elastogram with traditional ultrasound imagine. 
This allows the operator to visualize the liver while performing the elastography. Furthermore, 2DSWE-SSI has no upper limit and measurements can be obtained in patients with ascites. This makes the technique suited for patients with advanced disease, where portal hypertension is the main driver of prognosis. ${ }^{8}$ However, the studies investigating 2DSWE-SSI to diagnose portal hypertension and its severity have resulted in heterogeneous cut-off values, and the role of potential confounders has not been investigated.

We hypothesized that 2DSWE-SSI correlates with HVPG and can be used as a diagnostic marker of portal hypertension and oesophageal varices. We therefore performed an individual patient data meta-analysis to aggregate existing evidence. Our primary aim was to evaluate 2DSWE-SSI for ruling in and ruling out clinically significant portal hypertension in patients with cirrhosis, including subgroup and sensitivity analyses (compensated cirrhosis, patients without ascites, alcohol and viral aetiology, body mass index (BMI) below $25 \mathrm{~kg} / \mathrm{m}^{2}$, beta-blocker treatment and drinking status). Secondary aims were to assess the correlation between 2DSWE-SSI and the hepatic venous pressure gradient and evaluate the diagnostic accuracy of 2DSWE-SSI for severe portal hypertension and oesophageal varices.

\section{2 | METHODS}

This meta-analysis was based on a multicentre collaboration with data from individual patients in previously published studies. The study protocol was sent to all authors prior to data collection. Our report follows the preferred reporting items for systematic reviews and meta-analyses extension for diagnostic test accuracy studies (PRISMA-DTA) checklist. ${ }^{9}$ All included studies were approved by their local ethics board.

\section{1 | Studies}

We searched MEDLINE and Web of Sciences papers written in English that reported diagnostic studies with concurrent HVPG and 2DSWE-SSI measurements using the Supersonic Aixplorer system (Supersonic Imagine), in patients with cirrhosis. Last search update was 16 May 2019. We combined electronic searches with manual searches by scanning relevant reference lists. We invited corresponding authors to participate in this individual patient data metaanalysis. The search strategy and results can be seen in Table S1.

\section{2 | Participants}

We included patients for the meta-analysis if the following inclusion criteria were met: (a) 2DSWE-SSI performed within 3 weeks from the HVPG measurement; (b) 2DSWE-SSI performed with the patient in a fasting state; (c) cirrhosis diagnosed by standard clinical, biochemical, ultrasonographic, endoscopic and/or histologic characteristics; (d) age 18-80 years. We excluded patients in case of: (a) disseminated cancer disease; (b) hepatocellular carcinoma; (c) right-side cardiac failure NYHA class II-IV; (d) any decompensating event including severe infection in the interval between 2DSWE-SSI and HVPG measurement; (e) obstructive cholestasis evidenced by ultrasound; (f) hepatic inflammation evidenced by transaminase elevation of more than three times the upper limit of normal; and (g) transjugular intrahepatic portosystemic shunt.

\subsection{Index test and outcomes}

The index test was 2DSWE-SSI, reported in kilopascals ( $\mathrm{kPa})$. The description of the technique can be found elsewhere. ${ }^{10}$ The primary outcome was CSPH defined as HVPG $\geq 10 \mathrm{mmHg}$, measured by hepatic vein catheterization according to standard. ${ }^{11}$ Secondary outcomes were SPH defined as HVPG $\geq 12 \mathrm{mmHg}$, and oesophageal varices needing treatment assessed by endoscopy at the time of 2DSWE-SSI measurement. ${ }^{4}$

\subsection{Data extraction and quality assessment}

Authors who agreed to participate shared baseline characteristics, HVPG- and 2DSWE-SSI measurements for individual study participants. Shared data were compared to published results from the individual studies by $\mathrm{MBH}$ and $\mathrm{MT}$.

Four authors ( $\mathrm{MBH}, \mathrm{MT}, \mathrm{MI}$ and $\mathrm{AK})$ assessed study quality using the Quality Assessment of Diagnostic Accuracy Studies-2 (QUADAS-2) tool, which consists of four components: patient selection, index tests, reference standard and flow and timing (Figure 1). ${ }^{12}$ For each component, we labelled the risk of bias and applicability as low, high or unclear. We contacted authors for clarification, if papers had unclear risk components.

\subsection{Statistics}

We report summary statistics on pooled data as mean \pm SD and median $\pm I Q R$, depending on distribution. For between-group differences, we report Student's $t$ test in terms of normally distributed data, Wilcoxon rank sum test in terms of non-normally distributed data and Fisher's exact test in terms of proportions. The correlation between 2DSWE-SSI and HVPG was assessed by Pearson's correlation coefficient. We used area under the receiver operating characteristics (AUROC) curve statistics on pooled data to find the optimal 2DSWE-SSI cut-offs to rule out ( $90 \%$ sensitivity) and rule in (90\% specificity) CSPH, SPH and oesophageal varices. Cut-offs were rounded to the nearest integer. Next, we used the rule-in and rule-out cut-offs to create $2 \times 2$ tables for the distribution of true positives, false positives, false negatives and true negatives within each included study. Next, we used a meta-analytical diagnostic accuracy model, based on bivariate logistic regressions, to 


\begin{tabular}{|c|c|c|c|c|c|c|c|}
\hline & \multicolumn{4}{|c|}{ Risk of bias } & \multicolumn{3}{|c|}{ Applicability concerns } \\
\hline & 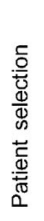 & 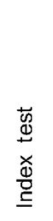 & 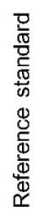 & 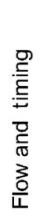 & 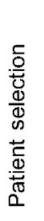 & 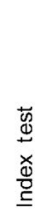 & 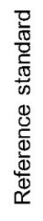 \\
\hline $\begin{array}{r}\text { Study } 1 \text { Multicenter Jansen } \\
2016, \text { Jansen 2017, Jansen } \\
2017 \\
\end{array}$ & 0 & 0 & 0 & 0 & 0 & $\mathrm{O}$ & $\mathrm{O}$ \\
\hline $\begin{array}{r}\text { Study } 2 \text { Korea Choi 2014, Kim } \\
\text { 2015, Lee 2016, Lee 2018, Lee } \\
2019 \\
\end{array}$ & 0 & $\mathrm{O}$ & $\mathrm{O}$ & $\mathrm{O}$ & 0 & $\mathrm{O}$ & $\mathrm{O}$ \\
\hline Study 3 Spain Procopet 2015 & 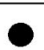 & 0 & 0 & 0 & 0 & 0 & 0 \\
\hline Study 4 Italy Stefanescu 2017 & 0 & 0 & 0 & 0 & 0 & $\mathrm{O}$ & $\mathrm{O}$ \\
\hline $\begin{array}{r}\text { Study } 5 \text { France Elkrief 2015, } \\
\text { Elkrief } 2018 \\
\end{array}$ & 0 & 0 & 0 & 0 & 0 & 0 & 0 \\
\hline
\end{tabular}

FIGURE 1 Quality assessment. Quality of the included studies according to the QUADAS-2 tool. The studies are assessed in terms of risk of bias and in terms of concerns regarding applicability. The index test is 2-dimensional real-time shear wave elastography by Supersonic Imagine, and reference standard is hepatic venous pressure gradient combine a hierarchical summary receiver operating characteristics (sROC) model and a bivariate random-effects model of sensitivity and specificity, visualized in SROC plots. ${ }^{13}$ We report $\mathrm{I}^{2}$ as a measure of heterogeneity between studies. An $\mathrm{I}^{2}$ value above $30 \%$ indicates substantial heterogeneity. We performed subgroup analyses on pooled data, owing to the low number of studies. Subgroup analyses included compensated cirrhosis, patients without ascites, alcohol and viral etiologies, and BMI below $25 \mathrm{~kg} / \mathrm{m}^{2}$. We also used pooled data to compare the diagnostic accuracy of 2DSWESSI with other non-invasive markers (Meld-Na, liver TE, spleen TE and liver stiffness-spleen size-to-platelet ratio risk score [LSPS]) using the DeLong test. We considered a P-value below 0.05 as statistically significant. We used STATA 15.0 for all analyses, with the MIDAS module for meta-analyses (Statacorp LP).

\section{3 | RESULTS}

\section{1 | Studies}

We identified 13 references referring to six studies which fulfilled the inclusion criteria of the meta-analysis and invited the corresponding authors to share individual patient data (see trial flow in Figure 2). Authors from five studies responded, ${ }^{14-25}$ while authors of one study did not reply. ${ }^{26}$ Characteristics of the five included studies can be seen in Table S1, and a list of excluded studies and cause for exclusion can be seen in Table S2. We included four single centre studies (Korea, France, Spain and Italy) and one multicentre study (Denmark, Germany and Belgium). ${ }^{14-25}$ Most patients were enrolled prospectively, ${ }^{16,18,22-25}$ with only one centre including patients enrolled both prospectively and retrospectively. ${ }^{17,19-21}$ For the quality assessment (Figure 1), we considered the included studies at low risk of bias for all domains, except regarding patient selection. We labelled all studies as having a high risk of bias for patient selection, because no study restricted inclusions to compensated cirrhosis patients only (for inclusion criteria in the individual studies, see Table S1). All studies have reported experienced 2DSWE-SSI operators with no more than three different operators per study (Table S1). Since portal hypertension is a key driver of hepatic decompensation, the inclusion of decompensated patients causes a high pretest risk of CSPH.

\subsection{Patients}

We received data on 519 participants of whom we included 328 for the individual patient data meta-analysis, while 191 met our exclusion criteria (Figure 2). The majority of patients had experienced at least one decompensating event prior to inclusion ( $n=237,73 \%$ ). Most patients were men (71\%), mean age was $56 \pm 10$ years and alcohol was the dominant cause of cirrhosis (53\%) (Table 1). Two hundred and sixty-eight (87\%) patients had CSPH. Compared to patients without CSPH, patients with CSPH had higher Meld-Na and ChildPugh scores and a higher proportion with ascites and decompensation at baseline (Table 1).

\section{3 | 2-dimensional real-time shear wave elastography to diagnose clinically significant portal hypertension}

Median 2DSWE-SSI liver stiffness was significantly higher for patients with $\mathrm{CSPH}$ (32.0 kPa, IQR [22.1-42.1]) compared to patients without CSPH (14.2 kPa, IQR [12.7-23.4]; $P$ < .001). The optimal cut-off to rule out CSPH was $14.0 \mathrm{kPa}$ (found by maximizing sensitivity to $90 \%$ ). At this cut-off, the sROC was 0.88 (0.85-0.91), summary sensitivity was $91 \%$ (86\%-94\%) and summary specificity was $37 \%$ (18\%-61\%) (Figure 3, Table 2). Of the 45 patients with 2DSWE-SSI below $14 \mathrm{kPa}$, 27 (60\%) were false negatives, as they did have HVPG $\geq 10 \mathrm{mmHg}$. We found that 21 (78\%) of the 27 false negative patients were decompensated at baseline. The optimal cut-off to rule in $\mathrm{CSPH}$ was $32.0 \mathrm{kPa}$ (found by maximizing specificity), corresponding to sROC, sensitivity 
FIGURE 2 Flowchart of study and patient selection. The five included studies were: (1) Multicentre (Denmark, Germany, Belgium), ${ }^{14-16}$ (2) Korea, ${ }^{17-21}$ (3) Spain, ${ }^{22}$ (4) Italy, ${ }^{23}$ (5) France. ${ }^{24,25}$ 2DSWE-SSI, 2-dimensional real-time shear wave elastography; HCC, hepatocellular carcinoma; HVPG, hepatic venous pressure gradient; ULN, upper limit of normal. ${ }^{\dagger}$ Two patients out of $328 \mathrm{had}$ unknown compensation status
605 references identified through Medline $(n=483)$,

Web of Science $(n=118)$ and reference lists $(n=4)$

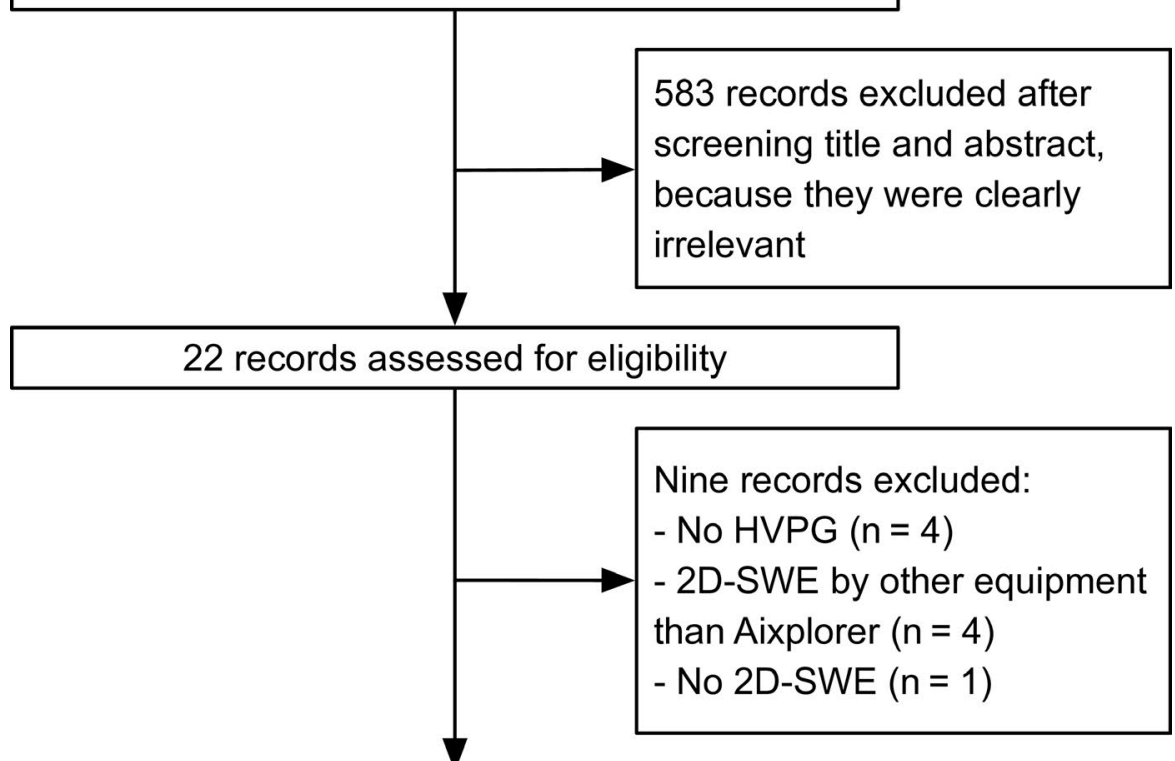

Nine articles, three letters and one abstract referring to six studies fulfilled the inclusion criteria and authors were invited to share individual patient data

Authors of one study did not reply to the invitation
Five studies from seven centers with a total of 519 patients were included for individual patient assessment

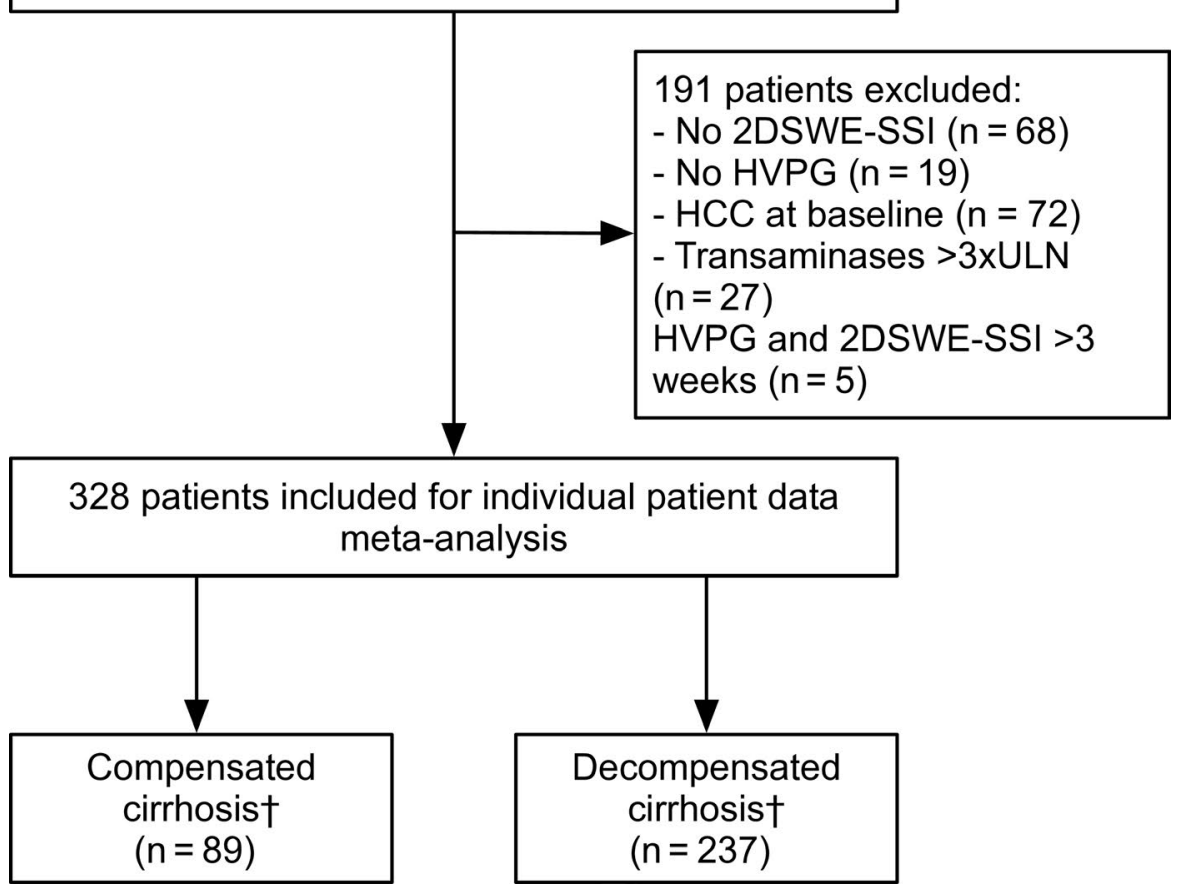




\begin{tabular}{|c|c|c|c|c|}
\hline & All & HVPG $<10 \mathrm{mmHg}$ & HVPG $\geq 10 \mathrm{mmHg}$ & \\
\hline & $328(100)$ & $42(13)$ & $286(87)$ & $P$-value \\
\hline Gender (male) & $233(71)$ & $31(74)$ & $202(71)$ & .720 \\
\hline Age (years) & $56 \pm 10$ & $56 \pm 13$ & $56 \pm 10$ & .973 \\
\hline $\begin{array}{l}\text { Compensated } \\
\text { cirrhosis }^{a}\end{array}$ & $89(27)$ & $30(71)$ & $59(21)$ & $<.001$ \\
\hline Meld-Na score ${ }^{a}$ & $13[9,18]$ & $9[7,13]$ & $14[10,19]$ & $<.001$ \\
\hline Child-Pugh score ${ }^{a}$ & $7[6,9]$ & $5[5,6]$ & $7[6,9]$ & $<.001$ \\
\hline $\begin{array}{l}\text { Ascites at } \\
\text { inclusion }^{a}\end{array}$ & $163(50)$ & $6(14)$ & $157(55)$ & $<.001$ \\
\hline Beta-blocker ${ }^{a}$ & $110(34)$ & $8(23)$ & $102(49)$ & .005 \\
\hline \multicolumn{5}{|l|}{ Measurements } \\
\hline 2DSWE-SSI (kPa) & $\begin{array}{l}30.4[19.3 \\
40.3]\end{array}$ & $14.2[12.7,23.4]$ & $32.0[22.1,42.1]$ & $<.001$ \\
\hline HVPG (mmHg) & $16[12,20]$ & $7[6,9]$ & $17[13,20]$ & $<.001$ \\
\hline \multicolumn{5}{|l|}{ Aetiology } \\
\hline NAFLD & $19(6)$ & $3(7)$ & $16(6)$ & .721 \\
\hline Alcohol & $173(53)$ & $13(31)$ & $160(56)$ & .003 \\
\hline Viral & $90(27)$ & $17(41)$ & $73(26)$ & .062 \\
\hline Other & $46(14)$ & $9(21)$ & $37(13)$ & .153 \\
\hline \multicolumn{5}{|l|}{ Metabolic risk factors } \\
\hline Excess drinking ${ }^{a}$ & $179(55)$ & $3(10)$ & $86(36)$ & .006 \\
\hline $\begin{array}{l}\text { Diabetes and/or } \\
\text { obesity }^{a}\end{array}$ & $121(37)$ & $17(49)$ & $104(50)$ & 1.00 \\
\hline BMI $\left(\mathrm{kg} / \mathrm{m}^{2}\right)^{\mathrm{a}}$ & $25[23,28]$ & $25[23,28]$ & $25[23,28]$ & .989 \\
\hline \multicolumn{5}{|l|}{ Blood tests } \\
\hline $\operatorname{ALT}(\mathrm{U} / \mathrm{L})^{\mathrm{a}}$ & $30[18,43]$ & $26[15,43]$ & $30[19,43]$ & .480 \\
\hline Albumin $(\mathrm{g} / \mathrm{L})^{\mathrm{a}}$ & $31[27,36]$ & $38[31,43]$ & $30[27,35]$ & $<.001$ \\
\hline $\begin{array}{l}\text { Bilirubin } \\
(\mu \mathrm{mol} / \mathrm{L})^{\mathrm{a}}\end{array}$ & $23[14,47]$ & $15[10,22]$ & $25[15,51]$ & $<.001$ \\
\hline$I_{N R}{ }^{a}$ & $1.2[1.1,1.4]$ & $1.0[0.9,1.3]$ & $1.2[1.1,1.4]$ & $<.001$ \\
\hline $\begin{array}{l}\text { Platelet count } \\
\left(10^{9} / \mathrm{L}\right)^{\mathrm{a}}\end{array}$ & $97[70,144]$ & $118[81,164]$ & $95[67,142]$ & .057 \\
\hline $\begin{array}{l}\text { Sodium } \\
(\mathrm{mmol} / \mathrm{L})^{\mathrm{a}}\end{array}$ & $\begin{array}{l}138[135, \\
141]\end{array}$ & $140[137,142]$ & $138[135,141]$ & $<.001$ \\
\hline
\end{tabular}

TABLE 1 Patient characteristics

Note: Values are presented as counts (\%), mean \pm SD or medians [interquartile range]. $P$-values are from Fisher's exact test for proportions, Student's $t$ test for means and Wilcoxon rank sum test for medians.

Abbreviations: 2DSWE-SSI, 2-dimensional real-time shear wave elastography by Supersonic Imagine; ALT, alanine aminotransferase; BMI, body mass index; HVPG, hepatic venous pressure gradient; INR, international normalized ratio of prothrombin time of blood coagulation; Meld-Na, model for end-stage liver disease with sodium NAFLD, non-alcoholic fatty liver disease.

${ }^{a}$ Missing data: two missing for decompensation, 111 for Meld-Na, 10 for Child Pugh score, 7 for ascites, 86 for beta-blocker, 58 for prior or current excess drinking, 86 for diabetes and/or obesity, 36 for BMI, 21 for ALT, 17 for albumin, 16 for bilirubin, 12 for INR, 12 for platelet count and 18 missing for sodium.

and specificity of $0.83(0.79-0.86), 47 \%$ (36\%-60\%) and $89 \%$ (74\%$96 \%$ ). Of the 150 patients with $2 \mathrm{DSWE}-\mathrm{SSI} \geq 32 \mathrm{kPa}$, five (3\%) were false positives, with HVPG $<10 \mathrm{mmHg}$. While sensitivities and specificities varied substantially between the five included studies, we did not detect evidence of heterogeneity, as $I^{2}$ was $0 \%-5 \%$ (Table 2). Use of the $14 \mathrm{kPa}$ and $32 \mathrm{kPa}$ cut-offs resulted in the following: 18 (5.5\%) true negative and 27 (8\%) false negative below $14 \mathrm{kPa}$; 145 (44\%) true positive and 5 false positive (1.5\%) above $32 \mathrm{kPa}$; and $136(41 \%)$ in the grey zone between 14 and $32 \mathrm{kPa}$. One hundred and seventeen of the 136 patients (86\%) in the grey zone had CSPH. 
(A)

Cut-off to rule out CSPH: 2DSWE-SSI < $14 \mathrm{kPa}$

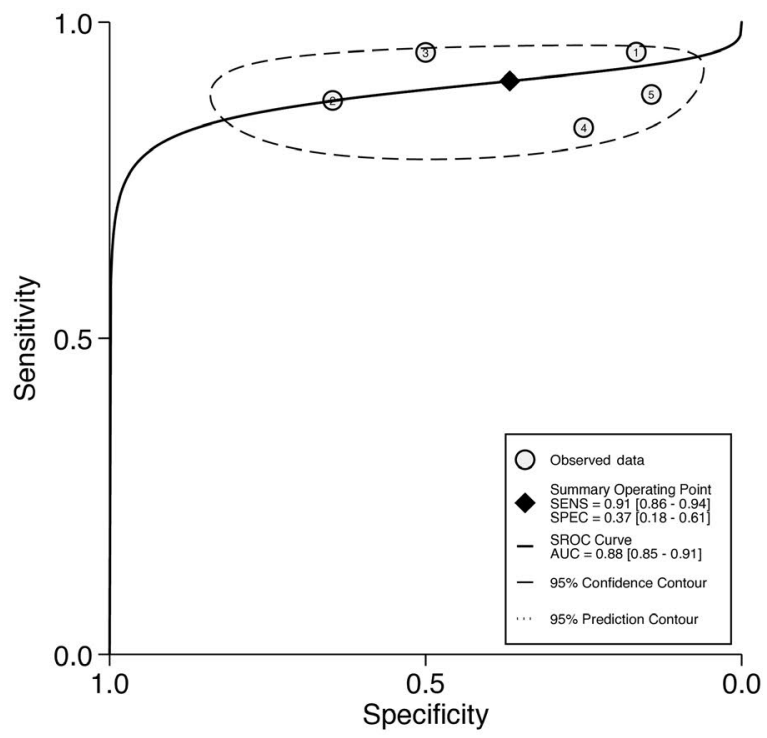

(C) Cut-off to rule out severe portal hypertension: 2DSWE-SSI < $18 \mathrm{kPa}$

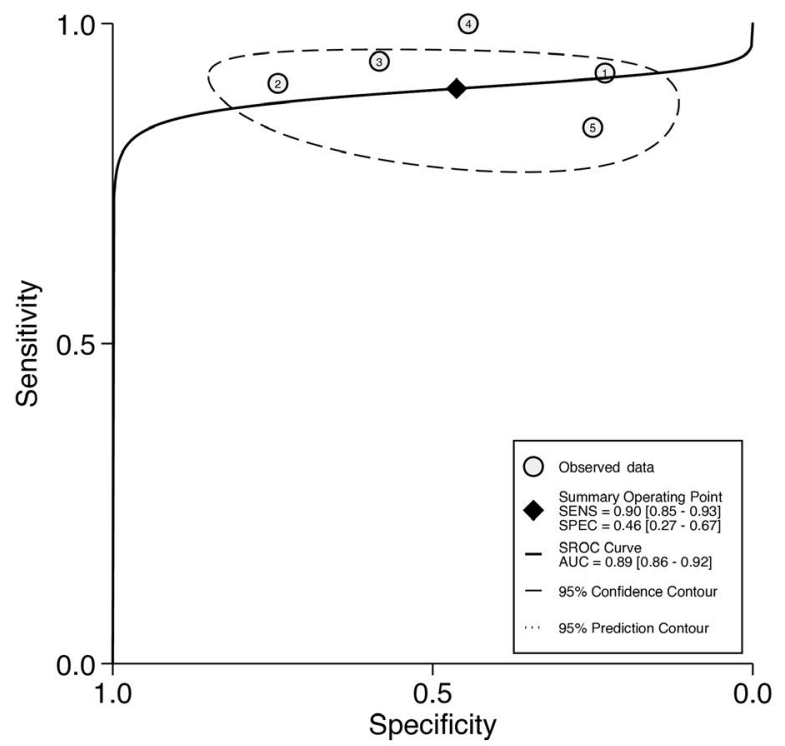

(B)

Cut-off to rule in $\mathrm{CSPH}$ : 2DSWE-SSI $\geq 32 \mathrm{kPa}$

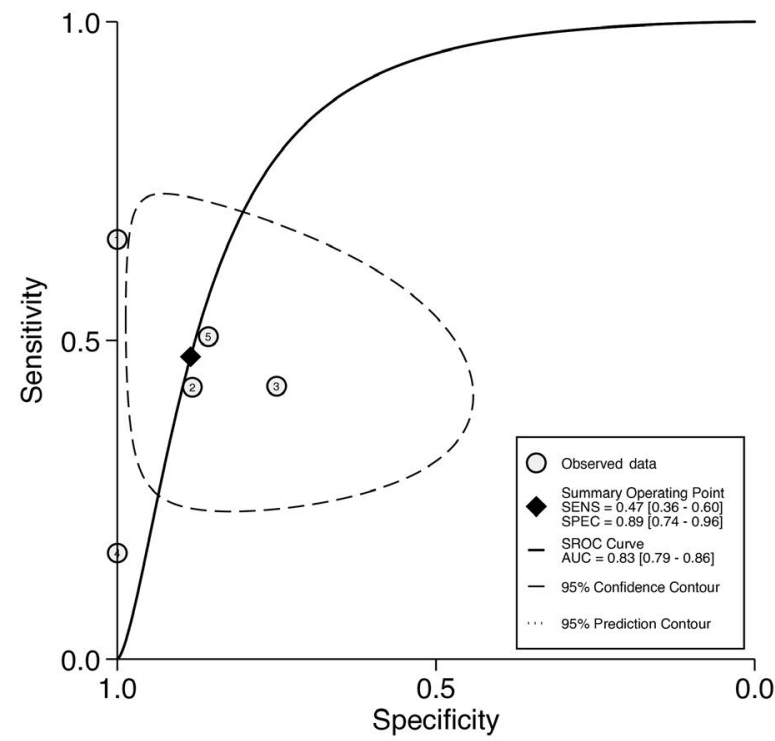

(D) Cut-off to rule in severe portal hypertension: 2DSWE-SSI $\geq 36 \mathrm{kPa}$

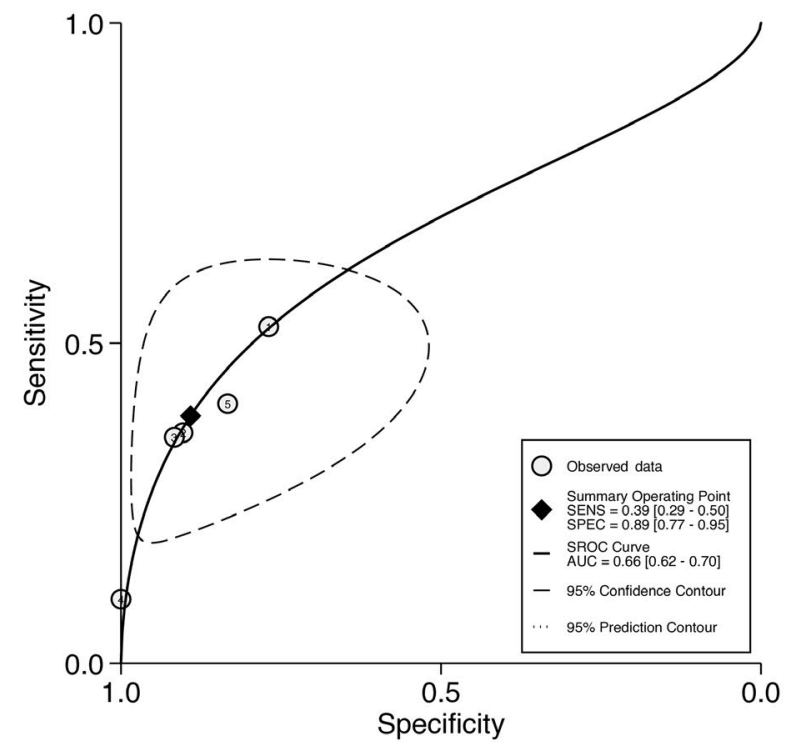

FIGURE 3 Hierarchical summary receiver operating characteristic plots of sensitivity and specificity. Hierarchical summary receiver operating characteristic plots (HSROC) of sensitivity and specificity using (A) a $14 \mathrm{kPa}$ cut-off for 2DSWE-SSI to rule out CSPH (derived by optimizing to $90 \%$ sensitivity in the pooled data). B, A $32 \mathrm{kPa}$ cut-off to rule in clinically significant portal hypertension (derived by optimizing to $90 \%$ specificity). C, A $18 \mathrm{kPa}$ cut-off to rule out severe portal hypertension. D, A $36 \mathrm{kPa}$ cut-off to rule in severe portal hypertension. The summary operating point presents the summary sensitivity and specificity encircled by a $95 \%$ prediction contour of the confidence interval. Individual studies are presented with a circle and a number: (1) Multicentre (Denmark, Germany, Belgium), (2) Korea, (3) Spain, (4) Italy, (5) France. 2DSWE-SSI, 2-dimensional real-time shear wave elastography by Supersonic Imagine; AUC, area under the summary receiver operating characteristic curve; CSPH, clinically significant portal hypertension; HSROC, hierarchical summary receiver operating characteristics curve; SENS, sensitivity; SPEC, specificity 
TAB LE 2 Diagnostic accuracy of 2-dimensional real-time shear wave elastography by Supersonic Imagine to rule out and rule in clinically significant portal hypertension, severe portal hypertension and oesophageal varices

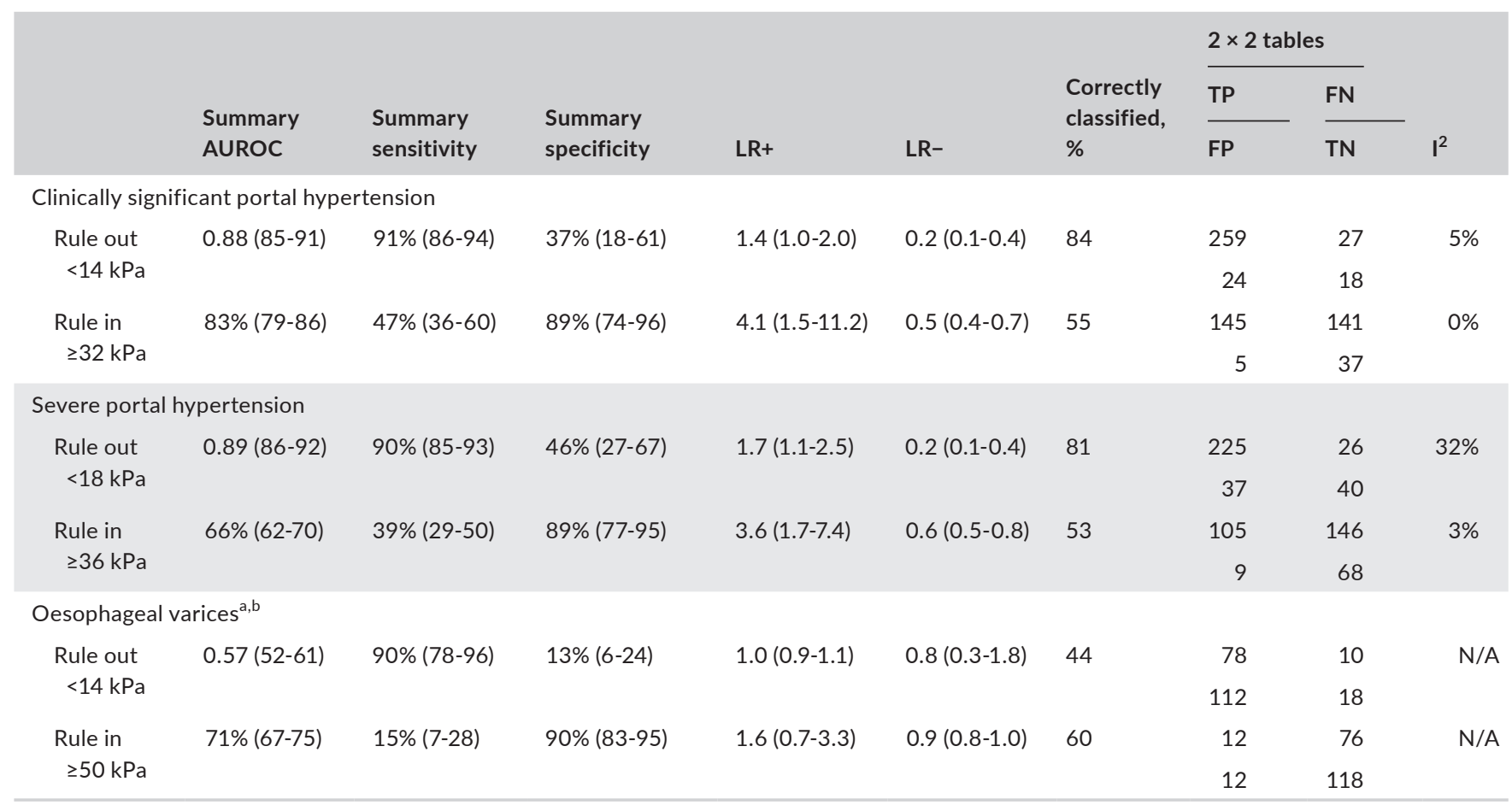

Note: We derived the 2DSWE-SSI rule out cut-offs by optimizing $90 \%$ sensitivity in the total cohort. Similarly, we derived the 2DSWE-SSI rule in cutoffs by optimizing to $90 \%$ specificity. Data are reported with $95 \%$ confidence interval in parenthesis

2DSWE-SSI, 2-dimensional real-time shear wave elastography by Supersonic Imagine; AUROC, area under the receiver operating characteristic curve; FN, false negative; FP, false positive; $I^{2}$, test for inconsistency between studies; kPa, kilopascal; LR-, negative likelihood; LR+ positive likelihood; TN, true negative; TP, true positive.

${ }^{a}$ Data on the presence of oesophageal varices were available for 218 patients with an endoscopy at baseline.

${ }^{\mathrm{b}}$ To perform the summary analysis, we replaced true positive, true negative, false negative and false positive containing zeros with 1.

We observed a significant correlation between 2DSWE-SSI and HVPG ( $r=0.458 P<.001)$ (Figure 4A). The correlation weakened for patients with HVPG $<10 \mathrm{mmHg}(r=0.221, P=.160)$, but improved for patients without ascites at baseline $(r=0.601, P<.001)$ (Figure 4B and 4C).

\section{4 | Subgroup analyses of 2-dimensional shear wave elastography by Supersonic Imagine to diagnose clinically significant portal hypertension}

Subgroup analyses showed that rule out CSPH at the cut-off $14 \mathrm{kPa}$ had similar sensitivity and higher specificity for patients with compensated cirrhosis, without ascites, viral aetiology or BMI $<25 \mathrm{~kg} /$ $\mathrm{m}^{2}$ (Table 3). We compared patients receiving beta-blockers ( $n=110$ ) versus those without beta-blockers ( $n=132$ ), and found that those on beta-blockers had significantly higher 2DSWE-SSI $(P=.002)$, higher rate of varices needing treatment $(P<.001)$, but not significantly higher MELD-Na $(P=.412)$. By ROC comparison, no significant difference was found between the diagnostic accuracy of 2DSWE-SSI in patients receiving beta-blockers versus those without beta-blocker treatment ( $P=.312$ for $\mathrm{CSPH} ; P=.589$ for $\mathrm{SPH}$ ). We had insufficient data on antiviral treatment to justify subgroup analyses. We compared abstinent $(n=130)$ and drinking patients $(n=96)$ and found no difference in liver stiffness $(P=.480)$ or diagnostic accuracy of 2DSWE-SSI ( $P=.864$ for CSPH; $P=.592$ for SPH).

To test the robustness of our sensitivity analyses taking into account that patients were from different studies, we performed a post-hoc multilevel logistic regression with study identifier as a random effect. This did not change the subgroup analyses (data not shown).

In a post-hoc analysis, we similarly did not find evidence of a correlation between 2DSWE-SSI and transaminases. The correlation coefficient between 2DSWE-SSI and ALT was 0.029, while the correlation coefficient for 2DSWE-SSI and AST was 0.146 . However, as we excluded patients with transaminases above three times the upper limit of normal, these results cannot be extrapolated to more extreme hepatic inflammation.

We did not have complete data on other non-invasive markers and scores for comparison with 2DSWE-SSI: Child-Pugh score ( $n=318$ available), TE of the liver ( $n=47$ available), LSPS ( $n=38$ available), Meld-Na score ( $n=310$ available) and TE of the spleen ( $n=99$ available). In the available data, 2DSWE-SSI was not different from Child-Pugh score, liver TE and LSPS for the diagnosis of CSPH 
FIGURE 4 Correlation plot.

Correlation scatter plots with fitted lines of 2DSWE-SSI and the hepatic venous pressure gradient. The Pearson correlation coefficient $(r)$ is reported in the upper left part of the graph. (A) Correlation between 2DSWE-SSI and HVPG for all 328 patients in the metaanalysis. (B) Correlation between 2DSWESSI and HVPG in the 55 patients without clinically significant portal hypertension (HVPG $\leq 10 \mathrm{mmHg}$ ). (C) Scatter plot of the correlation between 2DSWE-SSI and HVPG in 158 patients without ascites. 2DSWE-SSI, 2-dimensional real-time shear wave elastography by Supersonic Imagine; HVPG, hepatic venous pressure gradient
(A)

All patients

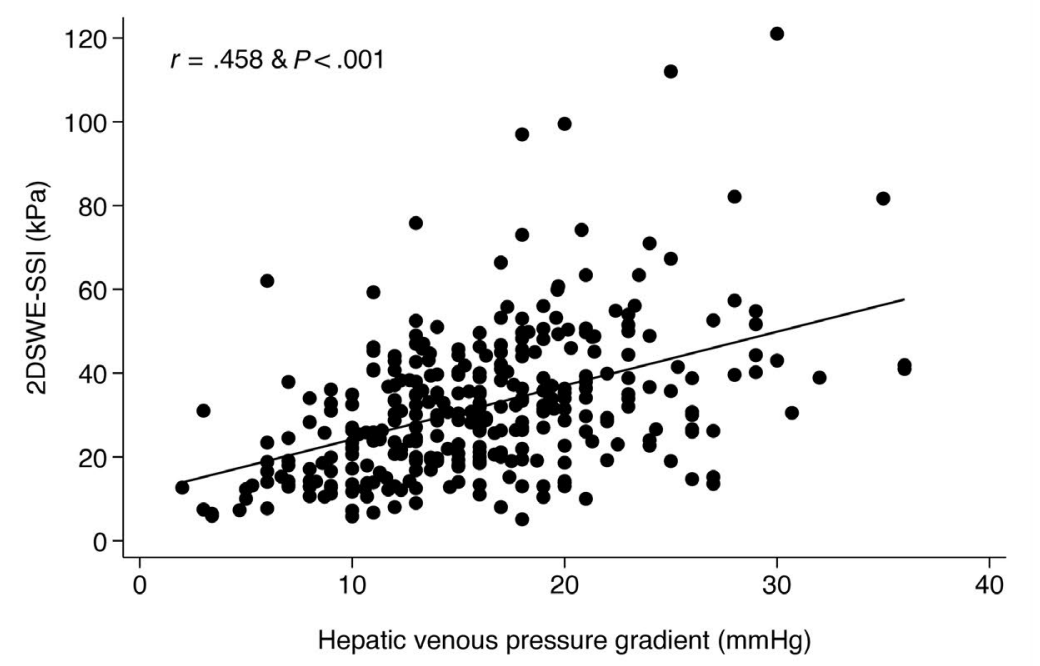

(B)

Patients with HVPG $<10 \mathrm{mmHg}$

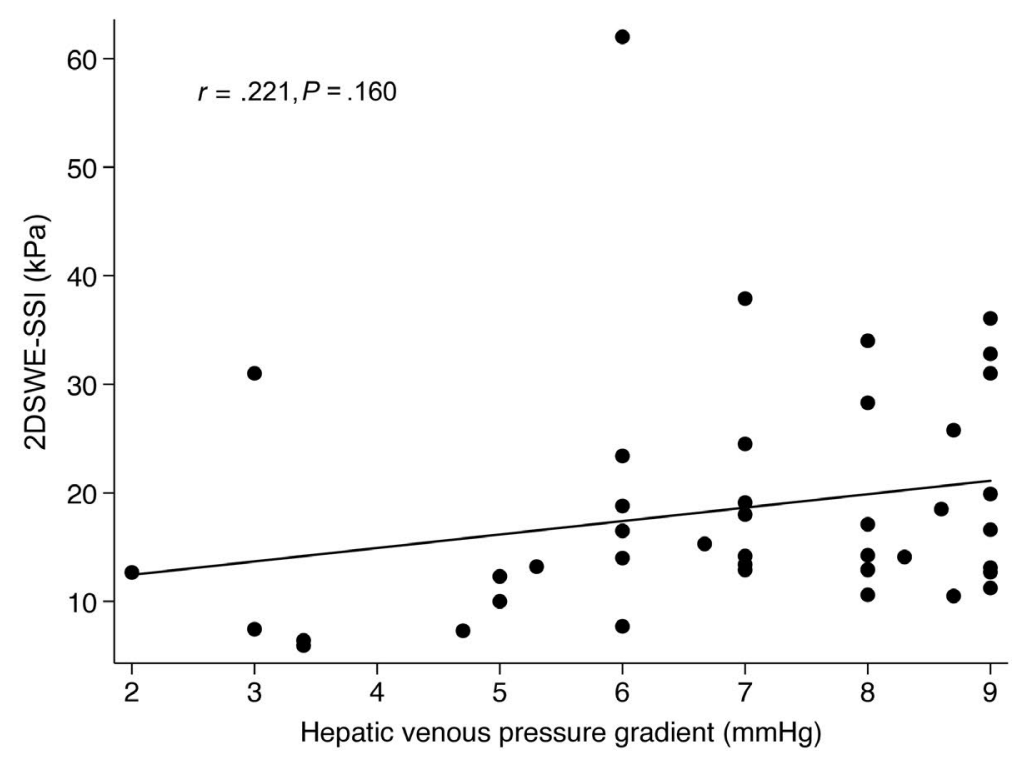

(C) Patients without ascites at baseline

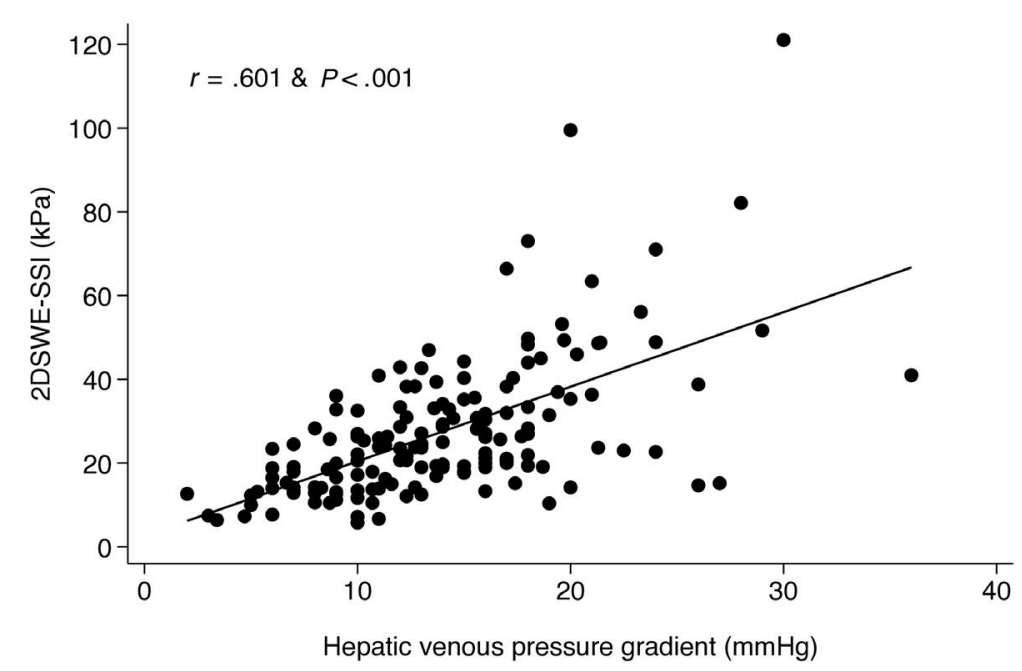


TAB LE 3 Subgroup diagnostic accuracy, sensitivity and specificity of 2-dimensional real-time shear wave elastography by Supersonic Imagine to rule out $(<14 \mathrm{kPa})$ and rule in $(\geq 32 \mathrm{kPa})$ clinically significant portal hypertension in patients with cirrhosis

\begin{tabular}{|c|c|c|c|c|c|c|}
\hline & AUROC & Sensitivity & Specificity & LR+ & LR- & $\begin{array}{l}\text { Correctly } \\
\text { classified, \% }\end{array}$ \\
\hline \multicolumn{7}{|c|}{ Compensated at baseline $(n=89)$} \\
\hline Rule in & $0.57(0.51-0.65)$ & $22 \%(12-35)$ & $93 \%(78-99)$ & $3.3(0.7-13.7)$ & $0.8(0.7-0.9)$ & 46 \\
\hline \multicolumn{7}{|c|}{ No ascites ( $n=158$ ) } \\
\hline Rule in & $0.65(0.59-0.71)$ & $36 \%(27-45)$ & $94 \%(81-99)$ & $4.8(1.0-22.3)$ & $0.7(0.5-1.0)$ & 49 \\
\hline \multicolumn{7}{|c|}{ Alcohol aetiology $(n=173)$} \\
\hline Rule out & $0.66(0.53-0.80)$ & $94 \%(90-97)$ & $39 \%(14-68)$ & $1.5(0.9-2.3)$ & $0.1(0.0-0.3)$ & 90 \\
\hline Rule in & $0.78(0.70-0.87)$ & $64 \%(56-72)$ & $92 \%(64-100)$ & $8.3(1.2-55.2)$ & $0.3(0.2-0.5)$ & 66 \\
\hline \multicolumn{7}{|c|}{$\mathrm{BMI}<25(\mathrm{n}=140)$} \\
\hline Rule out & $0.73(0.62-0.84)$ & $96 \%(91-99)$ & $50 \%(27-73)$ & $1.8(1.1-3.0)$ & $0.0(0.0-0.3)$ & 89 \\
\hline Rule in & $0.73(0.63-0.82)$ & $60 \%(51-69)$ & $85 \%(62-97)$ & $4.0(1.2-12.8)$ & $0.4(0.3-0.7)$ & 64 \\
\hline \multicolumn{7}{|c|}{ Beta-blocker treatment $(n=110)$} \\
\hline Rule out & $0.72(0.53-0.91)$ & $94 \%(88-99)$ & $50 \%(16-84)$ & $1.9(0.9-3.8)$ & $0.1(0.0-0.3)$ & 90 \\
\hline Rule in & $0.73(0.6-0.86)$ & $59 \%(49-69)$ & $88 \%(47-100)$ & $4.7(0.7-29)$ & $0.5(0.3-0.7)$ & 61 \\
\hline
\end{tabular}

Note: All subgroup analyses were performed on pooled data from the full cohort. We derived the 2DSWE-SSI rule out cut-offs by optimizing to $90 \%$ sensitivity and rule in cut-offs by optimizing to $90 \%$ specificity. All data are reported with $95 \%$ confidence interval in parenthesis.

Abbreviations: 2DSWE-SSI, 2-dimensional real-time shear wave elastography by Supersonic Imagine; AUROC, area under the receiver operating characteristic curve; BMI, body mass index; $\mathrm{I}^{2}$, test for inconsistency between studies; kPa, kilopascal; LR-, negative likelihood; LR+ positive likelihood; N, number; NA, not available.

(all $P>.05$ ), whereas 2DSWE-SSI outperformed Meld-Na and spleen TE $(P<.05)$ (Table S3). In post-hoc analyses, we also applied the Baveno VI criteria to 2DSWE-SSI instead of TE, to check how many patients had platelet count $>150 * 10^{9} / \mathrm{L}$ and 2DSWE-SSI $<14 \mathrm{kPa}$. This applied to only 7 of $316(2 \%)$ patients with a platelet count at baseline, of whom one had varices needing treatment.

\section{5 | 2-dimensional real-time shear wave elastography for severe portal hypertension and varices needing treatment}

A total of 251 (77\%) patients had SPH with HVPG $\geq 12 \mathrm{mmHg}$, and $88(40 \%)$ had varices needing treatment, in a subgroup of 218 patients with endoscopy at baseline. Patients with SPH had significantly higher 2DSWE-SSI than patients without SPH (33.4 kPa, IQR [23.7-44.1] vs 17.2 kPa, IQR [12.9-25.9]; $P$ < .001), but we found no significant difference in 2DSWE-SSI for patients with oesophageal varices compared to patients without oesophageal varices (32.9 $\mathrm{kPa}$, IQR [21.9-44.3] vs 29.3, IQR [19.9-39.4]; $P=$.124).

The optimal 2DSWE-SSI cut-off to rule out SPH was $18.0 \mathrm{kPa}$, and the optimal cut-off to rule in $\mathrm{SPH}$ was $36.0 \mathrm{kPa}$. For 2DSWESSI below $18 \mathrm{kPa}$, sROC was 0.89 (0.86-0.92), summary sensitivity was $90 \%$ (85\%-93\%) and summary specificity was $46 \%$ (27\%-67\%)
(Table 2) (Figure 3C and 3D). Of 66 patients with 2DSWE-SSI below $18 \mathrm{kPa}, 26$ (39\%) were false negative. For 2DSWE-SSI $\geq 36 \mathrm{kPa}$, sROC, summary sensitivity and specificity were 0.66 (0.62-0.70), $39 \%$ (29\%-50\%) and 89\% (77\%-95\%), respectively, with 9/114 (8\%) false positive. We found evidence of substantial between-study heterogeneity, as $\mathrm{I}^{2}$ was $32 \%$ for ruling out SPH at the $18 \mathrm{kPa}$ cutoff (Table 2). One-hundred and forty-eight patients (45\%) were in the grey zone of $18-36 \mathrm{kPa}$. We optimized two cut-offs, 14.0 and $50.0 \mathrm{kPa}$, to rule out and rule in varices needing treatment, according to sensitivity $=90 \%$ and specificity $=90 \%$ respectively. For $14.0 \mathrm{kPa}$, sROC was 0.57 (0.52-0.61), summary sensitivity was $90 \%(95 \% \mathrm{Cl}$ 78-96), summary specificity was $13 \%$ (95\% Cl 6-24) and 10/28 (36\%) were false negative. For $50.0 \mathrm{kPa}$, sROC, sensitivity, and specificity were 0.71 (0.67-0.75), 15\% (95\% Cl 7-28), and 90\% (95\% Cl 83-95), with $50 \%$ and false positives. However, use of these cut-offs left 166 (76\%) of patients in the grey zone between 14 and $50 \mathrm{kPa}$ (Table 2).

\section{4 | DISCUSSION}

In this meta-analysis based on data from five studies and 328 individual patients, we found that 2-dimensional shear wave elastography by Supersonic Imagine had a high sensitivity to rule out clinically significant portal hypertension at cut-off $14 \mathrm{kPa}$, but 
also a high false negative rate, as $60 \%$ of patients with 2DSWESSI below $14 \mathrm{kPa}$ had CSPH. The high false negative rate is likely caused by a high proportion of patients with decompensated disease. In contrast, only $3 \%$ of patients with 2DSWE-SSI above the rule in $\mathrm{CSPH}$ cut-off $32 \mathrm{kPa}$ were false positive. Furthermore, more than one-third of patients had 2DSWE-SSI between 14 and $31.9 \mathrm{kPa}$ and could therefore not be classified. We found an even higher proportion of patients in the grey zone between rule-out and rule-in cut-offs for severe portal hypertension (45\%) and varices needing treatment (74\%), which underlines the difficulty of designing decision-making tools for cirrhosis patients when the majority are decompensated.

We managed to include all but one published study on 2DSWESSI and HVPG. However, the results may be compromised by the limited sample size and a high risk of selection bias due to the high proportion of patients with decompensated cirrhosis. Furthermore, we could not test the influence of antiviral treatment due to insufficient data. We call for more studies that exclusively evaluate patients with compensated advanced chronic liver disease. The identification of cirrhosis patients with CSPH before decompensation is important according to a recent RCT, as those patients could benefit from primary prevention with non-selective beta-blockers. ${ }^{27}$ For this reason, it is unfortunate that existing studies primarily represent decompensated patients. In fact, the low number of compensated patients (27\%) in our study may also explain why subgroup analyses showed somewhat conflicting results: we found lower diagnostic accuracy in patients who were compensated at baseline, possibly due to a poor correlation between 2DSWE-SSI and HVPG in patients without CSPH. This finding is in contrast to studies investigating transient elastography for portal hypertension, where the strongest correlation between TE and HVPG is observed in patients with HVPG $<10 \mathrm{mmHg}^{7}$ Between-study heterogeneity cannot explain our findings, as $\mathrm{I}^{2}$ was low.

Currently, TE is the only elastography technique recommended by the Baveno group to identify $\mathrm{CSPH}$ and, in combination with platelet count, to rule out varices needing treatment. ${ }^{4}$ The Baveno $\mathrm{VI}$ criteria saves approximately $30 \%$ of variceal screening endoscopies in compensated cirrhosis patients. ${ }^{28}$ In our cohort, we did not find similar results for 2DSWE-SSI, as only $2 \%$ of patients had 2DSWE-SSI below $14 \mathrm{kPa}$ and platelet count above 150*10\%/ However, this discrepancy may not be due to differences between TE and 2DSWE-SSI, but rather due to the high proportion of decompensated patients in our pooled data, in whom variceal screening should always be done. Existing evidence suggests that 2DSWE-SSI and TE exhibit similar diagnostic accuracies for staging fibrosis across aetiologies. ${ }^{10,29}$ Two of the studies included in this meta-analysis similarly found no difference in diagnostic accuracy of the two techniques, albeit one study suggested that TE may have a higher failure rate than 2DSWE-SSI. ${ }^{22,24}$ Unfortunately, we did not have enough data for a robust meta-analytical comparison of TE and 2DSWE-SSI for portal hypertension. Therefore, our results should not be interpreted as a rejection of 2DSWE-SSI in favour of TE. Rather, new techniques are desirable to avoid monopolization.

Between-study variation in cut-off values is considered one of the main barriers for implementation of new diagnostic techniques. The individual patient data meta-analytical approach enabled us to derive consistent cut-offs from the pooled data, to be used in future studies. Another advantage was the ability to exclude patients who did not fulfil our inclusion and exclusion criteria, thereby limiting bias from patients with concomitant hepatic malignancy, severe liver inflammation or an extended time between 2DSWE-SSI and HVPG measurements.

In conclusion, this individual patient data meta-analysis is the largest series to date on 2-dimensional shear wave elastography by Supersonic Imagine for portal hypertension in patients with advanced liver disease. Our findings indicate that a cut-off of $14 \mathrm{kPa}$ may be tested in future studies including patients with compensated advanced chronic liver disease to rule out clinically significant portal hypertension.

\section{ACKNOWLEDGEMENTS}

The authors thank all patients and clinical researchers who were involved in the studies of this meta-analysis of individual patient data.

\section{CONFLICT OF INTEREST}

Juan Carlos García-Pagán (JCGP) received consultant fees from Shionogi, speaker fees from Gore and research grants from Novartis, Theravance and Exalenz. Fabio Piscaglia (FP) from an ESAOTE research contract. Bayer and GE, advisory board, Bracco and Bayer advisory board and speaker bureau. Remaining authors do not have any disclosures to report.

\section{AUTHOR CONTRIBUTIONS}

Maja Thiele (MT) designed the study, drafted the study protocol, contacted participating centres and collected data. Jonel Trebicka (JT), Annalisa Berzigotti (AB) and Aleksander Krag (AK) revised the study protocol. All authors contributed data to the study. Mie Balle Hugger (MBH) and MT performed data analyses. MBH, Mads Israelsen (MI) and MT performed the quality assessment of included studies and drafted the manuscript. All authors revised the manuscript and approved the final version.

\section{ORCID}

Maja Thiele (iD https://orcid.org/0000-0003-1854-1924

Mie B. Hugger iD https://orcid.org/0000-0003-4787-3565

Fabio Piscaglia iD https://orcid.org/0000-0001-8264-1845

Juan C. García-Pagán (iD https://orcid.org/0000-0001-9032-4954

Annalisa Berzigotti (iD https://orcid.org/0000-0003-4562-9016

Jonel Trebicka iD https://orcid.org/0000-0002-7028-3881

\section{REFERENCES}

1. Collaborators GDaH. Global, regional, and national disability-adjusted life-years (DALYs) for 333 diseases and injuries and healthy life expectancy (HALE) for 195 countries and territories, 
1990-2016: a systematic analysis for the Global Burden of Disease Study 2016. The Lancet. 2017;390(10100):1260-1344.

2. D'Amico G, Pasta L, Morabito A, et al. Competing risks and prognostic stages of cirrhosis: a 25-year inception cohort study of 494 patients. Aliment Pharmacol Ther. 2014;39(10):1180-1193.

3. Garcia-Tsao G, Groszmann RJ, Fisher RL, Conn HO, Atterbury CE, Glickman M. Portal pressure, presence of gastroesophageal varices and variceal bleeding. Hepatology. 1985;5(3):419-424.

4. de Franchis R. Expanding consensus in portal hypertension: report of the Baveno VI Consensus Workshop: stratifying risk and individualizing care for portal hypertension. J Hepatol. 2015;63(3):743-752.

5. Bosch J, Abraldes JG, Berzigotti A, Garcia-Pagan JC. The clinical use of HVPG measurements in chronic liver disease. Nat Rev Gastroenterol Hepatol. 2009;6(10):573-582.

6. Castera L, Chan HL, Arrese M, et al. EASL-ALEH clinical practice guidelines: non-invasive tests for evaluation of liver disease severity and prognosis. J Hepatol. 2015;63(1):237-264.

7. Colecchia A, Montrone L, Scaioli E, et al. Measurement of spleen stiffness to evaluate portal hypertension and the presence of esophageal varices in patients with HCV-related cirrhosis. Gastroenterology. 2012;143(3):646-654.

8. Dietrich C, Bamber J, Berzigotti A, et al. EFSUMB guidelines and recommendations on the clinical use of liver ultrasound elastography, update 2017 (Long Version). Ultraschall Med. 2017;38(4):e48.

9. Mclnnes MDF, Moher D, Thombs BD, et al. Preferred reporting items for a systematic review and meta-analysis of diagnostic test accuracy studies: the PRISMA-DTA statement. JAMA. 2018;319(4):388-396.

10. Herrmann E, Lédinghen VD, Cassinotto $C$, et al. Assessment of biopsy-proven liver fibrosis by 2D-shear wave elastography: an individual patient data based meta-analysis. Hepatology. 2018;67(1):260-272.

11. Silva-Junior $G$, Baiges $A$, Turon $F$, et al. The prognostic value of hepatic venous pressure gradient in patients with cirrhosis is highly dependent on the accuracy of the technique. Hepatology. 2015;62(5):1584-1592.

12. Whiting PF, Rutjes AW, Westwood ME, et al. QUADAS-2: a revised tool for the quality assessment of diagnostic accuracy studies. Ann Intern Med. 2011;155(8):529-536.

13. Dwamena B. MIDAS: module for meta-analytical integration of diagnostic accuracy studies. EconPapers. Statistical Software Components; 2007:s456880.

14. Jansen C, Bogs C, Verlinden W, et al. Algorithm to rule out clinically significant portal hypertension combining Shear-wave elastography of liver and spleen: a prospective multicentre study. Gut. 2016;65(6):1057-1058.

15. Jansen C, Bogs C, Krag A, Francque S, Trebicka J. Sequential shearwave elastography of liver and spleen rules out clinically significant portal hypertension in compensated advanced chronic liver disease. Gut. 2017;66(3):558-559.

16. Jansen C, Bogs C, Verlinden W, et al. Shear-wave elastography of the liver and spleen identifies clinically significant portal hypertension: a prospective multicentre study. Liver Int. 2017;37(3):396-405.

17. Choi S-Y, Jeong WK, Kim Y, Kim J, Kim TY, Sohn JH. Shear-wave elastography: a noninvasive tool for monitoring changing hepatic venous pressure gradients in patients with cirrhosis. Radiology. 2014:273(3):917-926.
18. Kim TY, Jeong WK, Sohn JH, Kim J, Kim MY, Kim Y. Evaluation of portal hypertension by real-time shear wave elastography in cirrhotic patients. Liver Int. 2015;35(11):2416-2424.

19. Lee C-M, Jeong WK, Lim S, et al. Diagnosis of clinically significant portal hypertension in patients with cirrhosis: splenic arterial resistive index versus liver stiffness measurement. Ultrasound Med Biol. 2016;42(6):1312-1320.

20. Lee JG, Sohn JH, Jeong JY, et al. Combined effect of hepatic venous pressure gradient and liver stiffness on long-term mortality in patients with cirrhosis. J Gastroenterol Hepatol. 2018;33:401-401.

21. Lee JG, Sohn JH, Jeong JY, et al. Combined effect of hepatic venous pressure gradient and liver stiffness on long-term mortality in patients with cirrhosis. Korean J Intern Med. 2020;35(1):88-98.

22. Procopet B, Berzigotti A, Abraldes JG, et al. Real-time shear-wave elastography: applicability, reliability and accuracy for clinically significant portal hypertension. J Hepatol. 2015;62(5):1068-1075.

23. Stefanescu H, Allegretti G, Salvatore V, Piscaglia F. Bidimensional shear wave ultrasound elastography with supersonic imaging to predict presence of oesophageal varices in cirrhosis. Liver Int. 2017;37(9):1405.

24. Elkrief L, Rautou P-E, Ronot M, et al. Prospective comparison of spleen and liver stiffness by using shear-wave and transient elastography for detection of portal hypertension in cirrhosis. Radiology. 2015;275(2):589-598

25. Elkrief L, Ronot $\mathrm{M}$, Andrade $\mathrm{F}$, et al. Non-invasive evaluation of portal hypertension using shear-wave elastography: analysis of two algorithms combining liver and spleen stiffness in 191 patients with cirrhosis. Aliment Pharmacol Ther. 2018;47(5):621-630.

26. Zhu Y-L, Ding H, Fu T-T, et al. Portal hypertension in hepatitis B-related cirrhosis: diagnostic accuracy of liver and spleen stiffness by 2-D shear-wave elastography. Hepatol Res. 2019;49(5):540-549.

27. Villanueva C, Albillos A, Genescà J, et al. Betablockers to prevent decompensation of cirrhosis in patients with clinically significant portal hypertension (PREDESCl): a randomised, double-blind, placebo-controlled, multicentre trial. Lancet. 2019;393(10181):1597-1608.

28. Maurice JB, Brodkin E, Arnold F, et al. Validation of the Baveno VI criteria to identify low risk cirrhotic patients not requiring endoscopic surveillance for varices. J Hepatol. 2016;65(5):899-905.

29. Thiele M, Detlefsen S, Sevelsted Møller L, et al. Transient and 2-dimensional shear-wave elastography provide comparable assessment of alcoholic liver fibrosis and cirrhosis. Gastroenterology. 2016;150(1):123-133

\section{SUPPORTING INFORMATION}

Additional supporting information may be found online in the Supporting Information section.

How to cite this article: Thiele M, Hugger MB, Kim Y, et al 2D shear wave liver elastography by Aixplorer to detect portal hypertension in cirrhosis: An individual patient data meta-analysis. Liver Int. 2020;00:1-12. https://doi. org/10.1111/liv.14439 